\title{
Familial risks in understanding type 1 diabetes genetics
}

\section{Kari Hemminki}

There have been impressive advances in the genetics of type 1 diabetes (T1D) that are increasingly being translated into a greater understanding of the disease mechanisms, as reviewed in this journal by Polychronakos and Li (Understanding type 1 diabetes through genetics: advances and prospects. Nature Reviews Genetics 12, 781-792) ${ }^{1}$. Each susceptibility gene confers a familial relative risk $(\lambda)$, and multiple susceptibility genes can be modelled to give a joint $\lambda$. The derived $\lambda$ can be compared to the empirical familial clustering of the disease as measured in epidemiological family studies that consider different family members: for example, parents and offspring or siblings. Sibling familial risk $\left(\lambda_{\mathrm{s}}\right)$ is often used in genetic studies. Polychronakos and Li gave $\lambda_{s}$ values for 34 loci that are reliably associated with T1D (Figure 1 of Polychronakos and $\mathrm{Li}^{1}$ ), and the authors concluded that the joint contribution of the identified loci was $\sim 60 \%$ of the empirical $\lambda_{\mathrm{s}}$ of 15 . We would like to raise concerns about the use of an empirical $\lambda_{s}$ of 15 for T1D and to highlight the wider need for empirical $\lambda_{\mathrm{s}}$ values to be regularly re-evaluated for other complex diseases.

The $\lambda_{s}$ of 15 for T1D has been used in other prominent studies and reviews without discussion of where it has come from ${ }^{1-3}$. In 2003, Hirschhorn ${ }^{4}$ cited a paper by Spielman and co-workers ${ }^{5}$ from 1980 as the origin of this value; this paper based $\lambda_{\mathrm{s}}$ on a literature review. As the incidence of T1D has increased rapidly, it is likely that a pre-1980 value for $\lambda_{\mathrm{s}}$ is no longer accurate. In 2009, we published a family study of T1D and other autoimmune diseases based on nationwide hospitalizations in Sweden between the years 1964 and 2007 (REF. 6). Practically all childhood patients with T1D are hospitalized in Sweden ${ }^{7}$. These data suggested that $\lambda_{\mathrm{s}}$ for T1D is about 12 for Swedes who were diagnosed in the mid-1990s. Using a $\lambda_{s}$ of 12 instead of 15 suggests that the joint gene effects actually account for a larger proportion of the familial clustering than was previously believed.

In summary, we recommend for T1D and other diseases that the effects of temporal changes in the empirical $\lambda_{\mathrm{s}}$ need to be monitored synchronously with genetic $\lambda_{s}$.

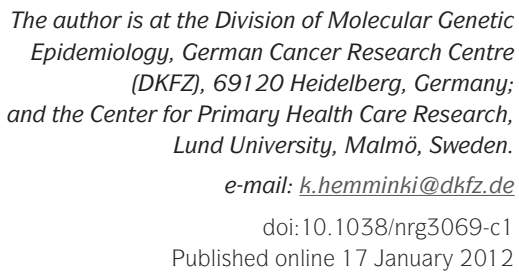

1. Polychronakos, C. $\& \mathrm{Li}, \mathrm{Q}$. Understanding type 1 diabetes through genetics: advances and prospects. Nature Rev. Genet. 12, 781-792 (2011).

2. The Wellcome Trust Case Control Consortium Genome-wide association study of 14,000 cases of seven common diseases and 3,000 shared controls. Nature 447, 661-683 (2007).

3. Todd, J. A. et al. Robust associations of four new chromosome regions from genome-wide analyses of type 1 diabetes. Nature Genet. 39, 857-864 (2007).

4. Hirschhorn, J. Genetic epidemiology of type 1 diabetes. Pediatr. Diabetes 4, 87-100 (2003).

5. Spielman, R. S., Baker, L. \& Zmijewski, C. M. Gene dosage and suceptibility to insulindependent diabetes. Ann. Hum. Genet. 44 135-150 (1980).

6. Hemminki, K., Li, X., Sundquist, J. \& Sundquist, K Familial association between type 1 diabetes and other autoimmune and related diseases. Diabetologia 52, 1820-1828 (2009).

7. Ludvigsson, J. F., Ludvigsson, J., Ekbom, A. \& Montgomery, S. M. Celiac disease and risk of subsequent type 1 diabetes: a general population cohort study of children and adolescents. Diabetes Care 29, 2483-2488 (2006).

\section{Acknowledgements}

This work was supported by Deutsche Krebshilfe, ALF grants of Region Skane and EU FP7/2007-2013 grant 260715.

Competing interests statement

The authors declare no competing financial interests. 